\title{
Electromagnetic controllable surfaces based on trapped-mode effect
}

\author{
V. Dmitriev ${ }^{1}$, S. Prosvirnin ${ }^{2,3}$, V. Tuz ${ }^{2,3^{*}}$, and M. N. Kawakatsu ${ }^{1}$ \\ ${ }^{1}$ Department of Electrical Engineering, Federal University of Para, Belem, Para, Brazil \\ ${ }^{2,3}$ Institute of Radioastronomy of National Academy of Sciences of Ukraine \\ ${ }^{2,3}$ School of Radio Physics, Karazin Kharkiv National University, Ukraine \\ *corresponding author, E-mail: tvrerian. kharkov.ua
}

\begin{abstract}
In this paper we present some recent results of our theoretical investigations of electromagnetically controllable surfaces. These surfaces are designed on the basis of periodic arrays made of metallic inclusions of special form which are placed on a thin substrate of active material (magnetized ferrite or optically active semiconductor). The main peculiarity of the studied structures is their capability to support the trapped-mode resonance which is a result of the antiphase current oscillations in the elements of a periodic cell. Several effects, namely: tuning the position of passband and the linear and nonlinear (bistable) transmission switching are considered when an external static magnetic field or optical excitation are applied. Our numerical calculations are fulfilled in both microwave and optical regions.
\end{abstract}

\section{Introduction}

The development of smart materials in microwave and optical regions can be realized by using plasmonic and metamaterial technologies. Here the promising way is to combine some known active materials with special artificial inclusions which are the key elements of metamaterial constructions [1-4]. As an example, a metamaterial array can be placed on the magnetic (ferrite, antiferromagnetic) or semiconductor substrate. In case of magnetic substrates, properties tuning can be realized by an external static magnetic field (ESMF). Also the conception of controlling the light propagation with light which is based on the nonlinear effect of optical bistability or multistability can be employed in the optical region. The devices based on these principles can have numerous applications in microwave and optical switching, differential amplification, optical transistor, power limiting, pulse shaping and optical digital data processing functions.

The goal of this paper is to present the peculiarity of linear and nonlinear microwave and optical tuning and switching in a planar metamaterial which bears the trappedmode resonances. We consider a planar metamaterial made of an array of double-ring (DR) elements placed on a thin magnetic or semiconductor substrate (Fig.1). Such configuration is chosen due to the fact that DR elements are polarization insensitive at the normal incidence of the exciting wave which can enlarge the area of potential applications.

Thus, we demonstrate that by using a magnetized ferrite substrate in this array it is possible to tune the trapped-mode frequency with external magnetic field. Our calculations show that in the microwave region, when ESMF is applied, the shift can reach $20 \%$ of the resonant frequency. The orientation of ESMF can be different, namely normal to the plane (Faraday geometry) of the substrate or in-plane. Note that in the latter case the system becomes polarization dependent.

Switching of the transmission band with the ESMF can be achieved in the array placed on a magnetized ferrite substrate in the case when the frequency of the trappedmode resonance is tuned nearly the frequency of the ferromagnetic resonance. We show that for the magnetization applied in the Faraday geometry, a good electromagnetic switching can be realized.

Another possibility of controlling electromagnetic radiation is to use a semiconductor substrate. We show the possibility to almost completely switch off the array transmission band by optically activating its silicon substrate. For low plasma density no effect is produced on the array responses. However, increasing plasma density above a certain value the structure becomes almost totally reflective as a consequence of the significant increase of the real and imaginary parts of the refraction index.

Still another possibility of controlling the electromagnetic wave propagation is based on the effect of optical bistability. Typically the devices realized on the optical bistability principle consist of a resonant cavity containing a material which refraction index or/and absorption coefficient are depended on the field intensity of incident light. A promising way to reduce the sizes of such devices and the input field intensity required for optical bistable switching lies in using planar metamaterials which can support the trapped-mode resonances.

The strong field confinement and nonlinear effects enhancement is achieved in the investigated arrays by using a nonlinear substrate made of antiferromagnetic film. The system is considered to be under ESMF applied in the Faraday geometry. In the structures of such kind, it is possible to obtain sufficient field localization in a thin nonlinear substrate which leads to realization of all-optical 
switching. In particular we show that the switching can be achieved between different levels of transmission near the frequency of the trapped-mode excitation.

\section{Problem statement}

The square unit cell $\left(d_{x}=d_{y}\right)$ of the structure under study consists of one double-ring (DR) resonator (Fig. 1). The radii of the outer and inner rings are $a_{1}$ and $a_{2}$, respectively. The width of both metal rings is $2 w$. The array is placed on a dielectric substrate with the thickness $h$.

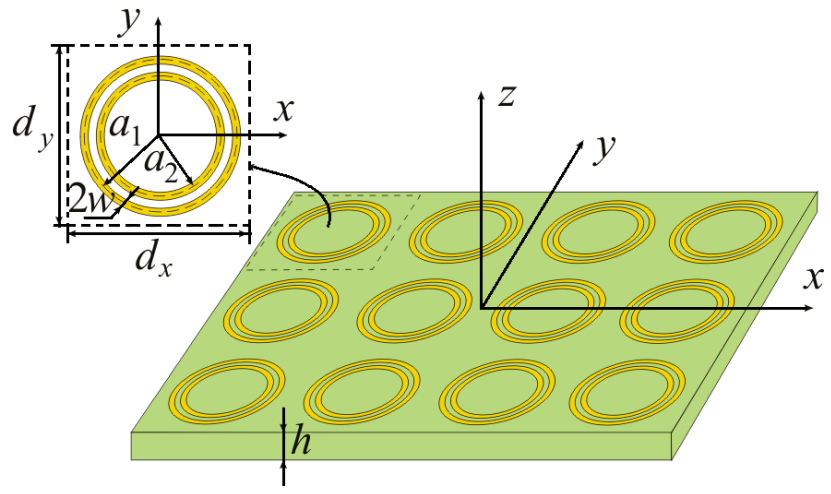

Figure 1. Array of two concentric metal rings on a substrate made of active material.

As the excitation field, the normally incident linearly polarized plane monochromatic wave of frequency $\omega$ and amplitude $A$ is selected.

To provide the numerical calculations we use both our own algorithms and software and a commercial one. The first technique is based on the Method of Moments $[5,6]$. It involves solving an integral equation for surface currents induced in the metallic pattern by the incident electromagnetic wave and calculating the scattered fields produced by currents as a superposition of partial spatial waves. The Method of Moment is paired with a special algorithm which allows solving the nonlinear problem $[2,3]$. As commercial software we use Computer Simulation Technology (CST) Studio [7].

\section{Numerical results}

\subsection{Array on ferrite substrate}

In [2], a parametric study of the DR array placed on a dielectric substrate was made in order to optimize the geometrical parameters and to obtain high quality factor $(Q-$ factor) trapped-mode resonant conditions. In this array, the trapped-mode corresponds to a narrow transmission resonance which exists between two broad reflection resonances. The latter resonances are due to the two individual resonances of the rings. For the substrate with losses, a compromise exists between high $Q$-factor and the maximum transmittance at the trapped-mode resonance. These parameters are about 12 and $70 \%$, respectively. The high $Q$-factor is obtained when the rings are close to each other and are very narrow. However, in this case the maximum transmittance is reduced, since the loss is increased due to higher field confinement between the rings. In order to obtain a high $Q$-factor and a high transmittance at the trapped-mode resonance, we use the following geometrical parameters (see Fig. 1): $d_{x}=d_{y}=13, a_{1}=6, a_{2}$ $=5,2 w=0.2$ and $h=1.6$ (all dimensions are in millimeters).

For the array placed on a ferrite substrate we consider the mathematical model of the magnetized ferrite substrate described in [8]. In the following we show the possibility to shift the trapped-mode resonant frequency with magnetization of the ferrite substrate. The chosen ferrite material is TTI-3000 [8]. The ferromagnetic resonant frequency of $\omega_{0} / 2 \pi$ can be controlled by the ESMF $\mathbf{H}_{0}$.

The reflection and transmission spectra of this structure are shown in Fig. 2 for magnetization in $x$ - and $y$-directions, and in Fig. 3 for $z$-direction. For comparison, in these figures the structure responses for the array with nonmagnetized ferrite substrate are also shown.

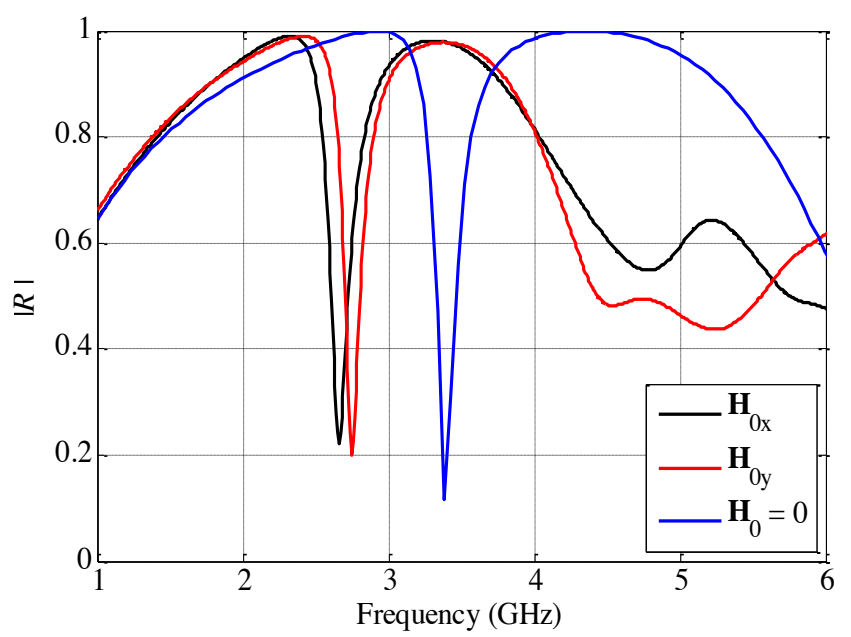

(a)

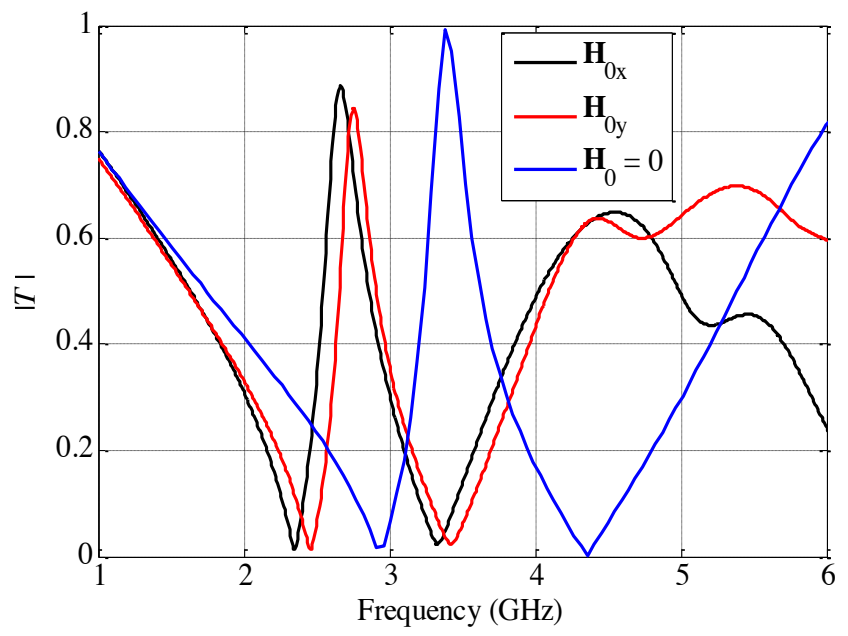

(b)

Figure 2. Reflection (a) and transmission (b) spectra of array placed on ferrite substrate, $\omega_{0} / 2 \pi=3,5 \mathrm{GHz}$. 


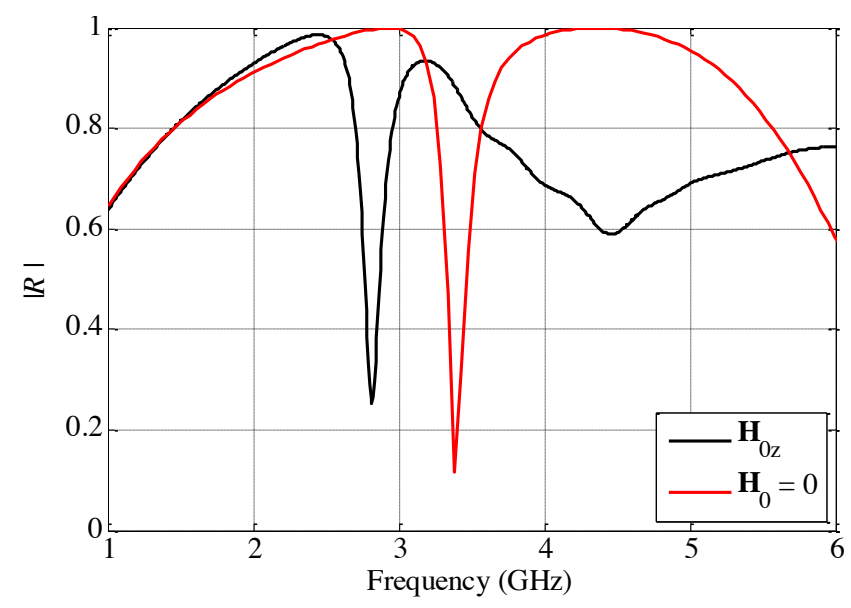

(a)

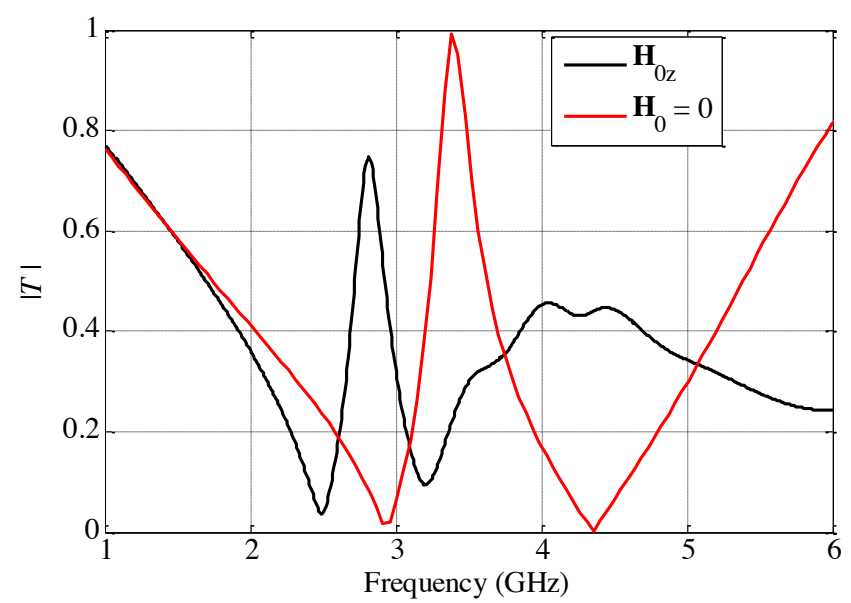

(b)

Figure 3. Reflection (a) and transmission (b) spectra of the array placed on a ferrite substrate magnetized in $z$ direction and for a non-magnetized one; $\omega_{0} / 2 \pi=3,5 \mathrm{GHz}$.

As can be seen in Figs. 2 and 3, a similar resonant frequency shift is achieved for different orientation of the magnetization. This shift is about $20 \%$ of the resonant frequency. However, for the normal magnetization the attenuation in the maximum of transmittance is larger than for parallel one. Note that for parallel magnetization the structure is polarization dependent, while for normal one it is polarization independent (as explained in [2]), but the dependence on polarization is small in the trapped-mode region, as can be observed in Fig. 2, where we show the cases of magnetization parallel $\left(\mathbf{H}_{0 x}\right)$ and orthogonal $\left(\mathbf{H}_{0 y}\right)$ with respect to the incident wave polarization.

To analyze the effect of the ferromangetic resonant frequency $\omega_{0}$ on the trapped-mode band, we show in Fig. 4 the structure responses for magnetization in $z$-direction for different values of $\omega_{0}$. One can see that near the ferromagnetic resonance frequency, the trapped-mode resonance shift is increased, however the attenuation in the maximum of transmittance is also increased. This is an obvious consequence of the ferromagnetic resonant characteristics because near the resonance the values of both the real and imaginary parts of the permeability tensor parameters are larger and their variation is higher [1].

When the magnetization is along $z$-direction, the Faraday and Kerr effects take place in the ferrite substrate. The polarization state of the electromagnetic waves reflected from and transmitted through the structure can be analyzed using the azimuth $\theta$ and ellipticity $\eta$ angles, as illustrated in the inset of Fig. 5b [1]. These angles were calculated for $\omega_{0} / 2 \pi=3,5 \mathrm{GHz}$ and are shown in Fig. 5. As it can be seen, in the trapped-mode band the Faraday rotation enhancement provided by the structure is about 2 times in comparison with the ferrite substrate without metallization.

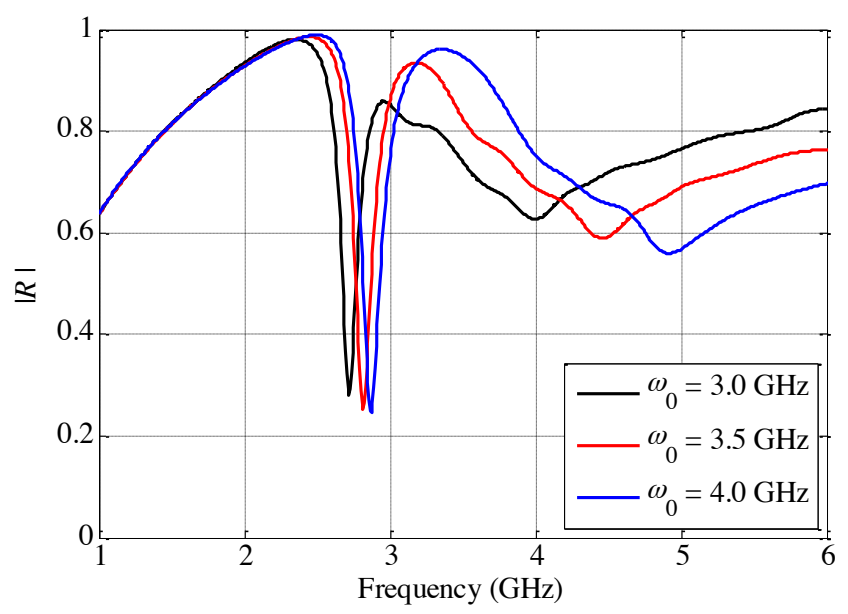

(a)

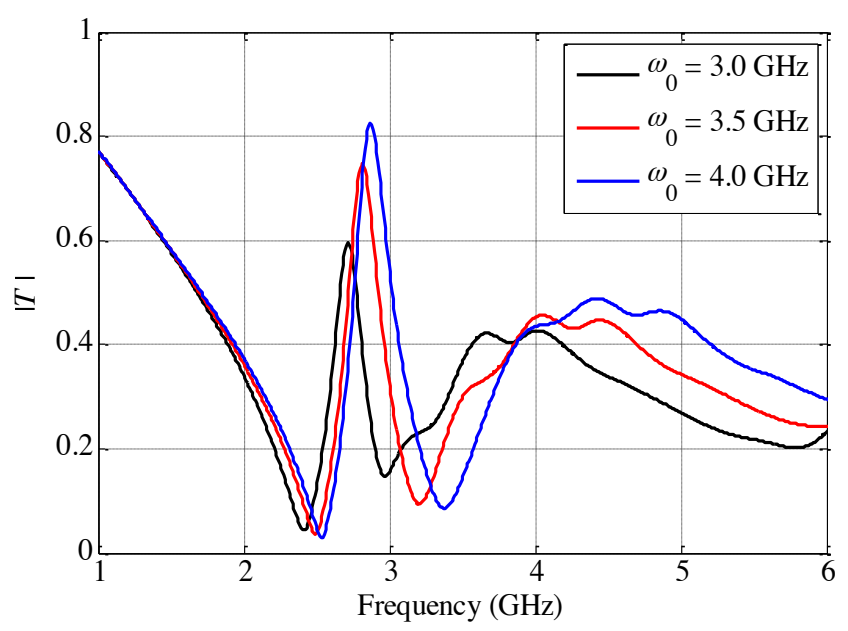

(b)

Figure 4. Reflection (a) and transmission (b) spectra of the array placed on a ferrite substrate magnetized in $z$ direction for different $\omega_{0}$.

Since in the vicinity of the ferromagnetic resonance the magnetic losses are very large, we investigate the possibility of designing the array with switch on and off functionality of the trapped-mode transmission band by adjusting the ferromagnetic resonant frequency. In order to show this possibility, we considered the substrate made of ferrite TT2- 
125 with $\omega_{0} / 2 \pi=3,5 \mathrm{GHz}$. In Fig. 6 the responses of the structure are shown for the magnetized ferrite substrate and for the non-magnetized one. As can be seen, the transmission coefficient can be switched from 1 to 0.3 at the trapped-mode resonance. We believe that by optimization of the geometrical and physical parameters, it is possible to achieve a more effective switching.

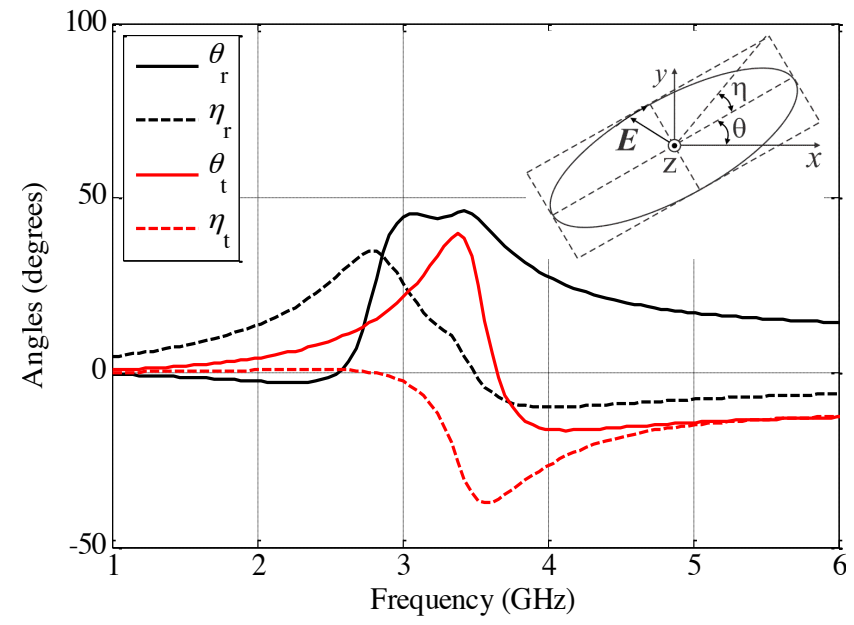

(a)

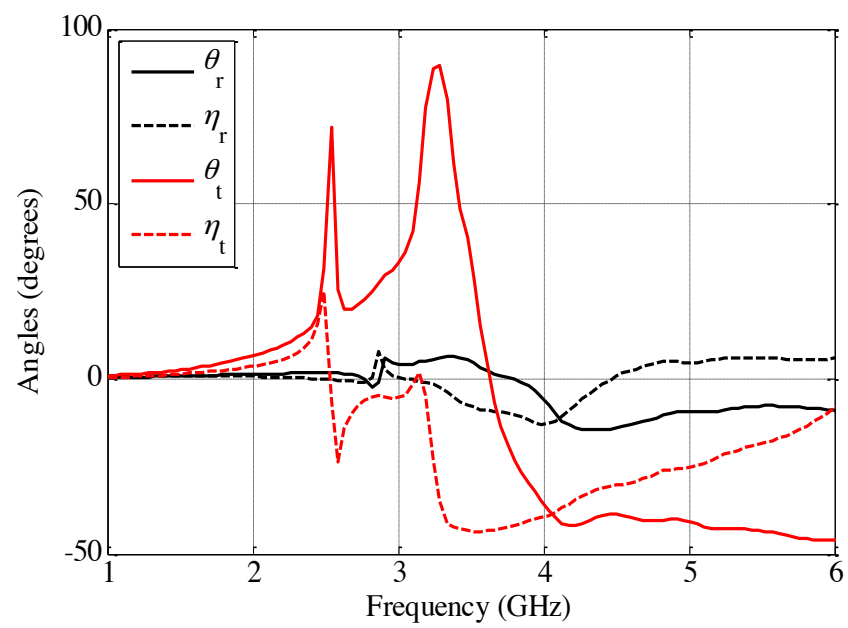

(b)

Figure 5. Azimuth $\theta$ and ellipticity $\eta$ angles of waves reflected from and transmitted through ferrite substrate without metalization (a) and through the structure with magnetization in $z$-direction; $\omega_{0} / 2 \pi=3,5 \mathrm{GHz}$.

\subsection{Array on semiconductor substrate}

For the array placed on a silicon substrate we consider the mathematical model of an optically activated silicon substrate described in [9]. In Fig. 7, the array responses for different values of the plasma density $N$ are shown. For $N=$ $10^{12} \mathrm{~cm}^{-3}$, the influence of induced plasma on the refraction index of the silicon substrate is negligible and a high $Q$ factor transmission resonance exists with almost total transmission at $40 \mathrm{GHz}$. Increasing $N$ to $10^{18} \mathrm{~cm}^{-3}$, one comes to the regime of almost complete reflection of the incident wave due to the significant increase of the real and imaginary parts of the silicon refraction index.

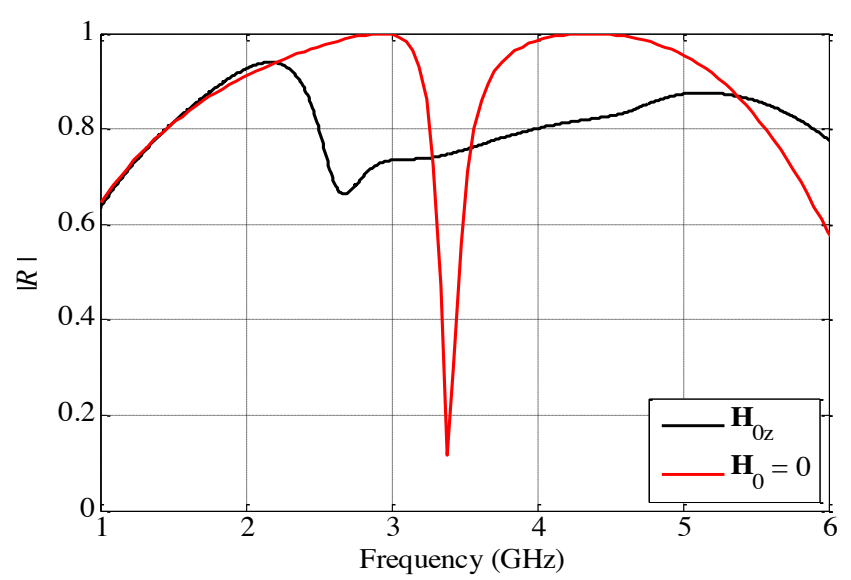

(a)

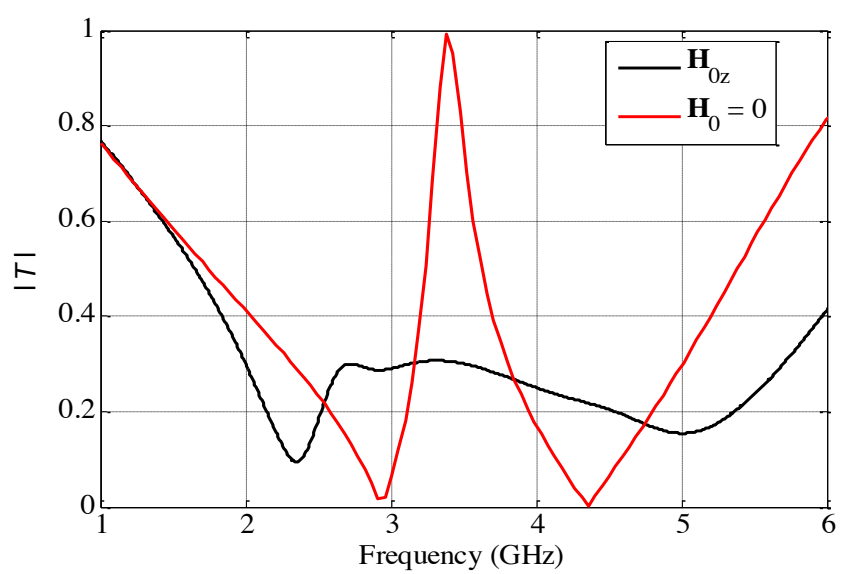

(b)

Figure 6. Reflection (a) and transmission (b) spectra of the array placed on a ferrite substrate magnetized in $z$ direction, and for a nonmagnetized one. The ferrite material is TT2-125 with $\omega_{0} / 2 \pi=3,5 \mathrm{GHz}$.

\subsection{Array on nonlinear antiferromagnetic substrate}

Finally, we consider a situation when the DR array is placed on a substrate made of antiferromagnetic film (AF). The metamaterial parameters are $d_{x}=d_{y}=0.3, a_{1}=0.11, a_{2}$ $=0.09,2 w=0.004$ and $h=0.05$ (all dimensions are in millimeters). As a material for the substrate, $\mathrm{MnF}_{2}$ antiferromagnetic film is considered [10,11]. The ESMF is applied to the system in the Faraday geometry. The physical parameters of the AF film are $\varepsilon_{\mathrm{r}}=5.5, H_{e}=450 \mathrm{kG}, H_{a}=$ $7.87 \mathrm{kG}$ and $M_{0}=0.6 \mathrm{kG}$, the gyromagnetic ratio is $\gamma=$ $1.97 \times 10^{10} \mathrm{rad} . \mathrm{s}^{-1} \cdot \mathrm{kG}^{-1}$ and the damping coefficient is fixed at $\tau=4.25 \times 10^{-4}$. For the fixed external magnetic field $\mathbf{H}_{0}=$ $1.0 \mathrm{kG}$, in the dispersion curves of the permeability tensor components there are two resonant frequencies, $\mathfrak{x}_{1} \approx 0.262$ ( $\mathrm{f} \approx 0.2618 \mathrm{THz})$ and $\mathfrak{x}_{2} \approx 0.268(\mathrm{f} \approx 0.2678 \mathrm{THz})$ which 
are shown in Fig. 8. Here $\mu_{1}=1+\chi_{1}$ and $\mu_{2}=\chi_{2}$ are diagonal and nondiagonal elements of the permeability tensor, and the expressions of magnetic susceptibility $\chi_{1}=\chi_{x x}^{(1)}=\chi_{y y}^{(1)}$ and $i \chi_{2}=\chi_{x y}^{(1)}=-\chi_{y x}^{(1)}$ can be found in $[10,11]$. Under the action of intense light the dynamical magnetization in AF media is coupled nonlinearly with the wave magnetic field which leads to the magnetic optical nonlinearity. In this case the magnetic susceptibility can be entered as follows:

$$
\chi_{i k}^{(1)}(\mathbf{H})=\chi_{i k}^{(1)}+\sum_{j=1}^{3} \chi_{i k j}^{(2)} H_{j}+\sum_{j=1}^{3} \sum_{m=1}^{3} \chi_{i k j m}^{(3)} H_{j} H_{m},
$$

where the expressions and typical dispersion curves for the second- and third-order susceptibility $\left(\chi_{i k j}^{(2)}, \chi_{i k j m}^{(3)}\right)$ are given in $[10,11]$. In the vicinity of each resonant frequency, the real and imaginary parts of these susceptibility components are of the order $10^{-8}$ and they are symmetrical and antisymmetrical with respect to the resonant frequencies, respectively.

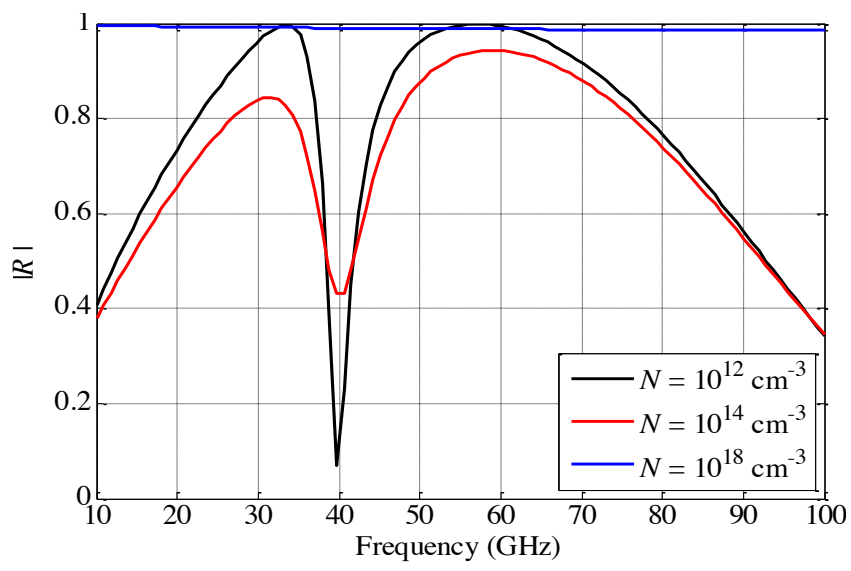

(a)

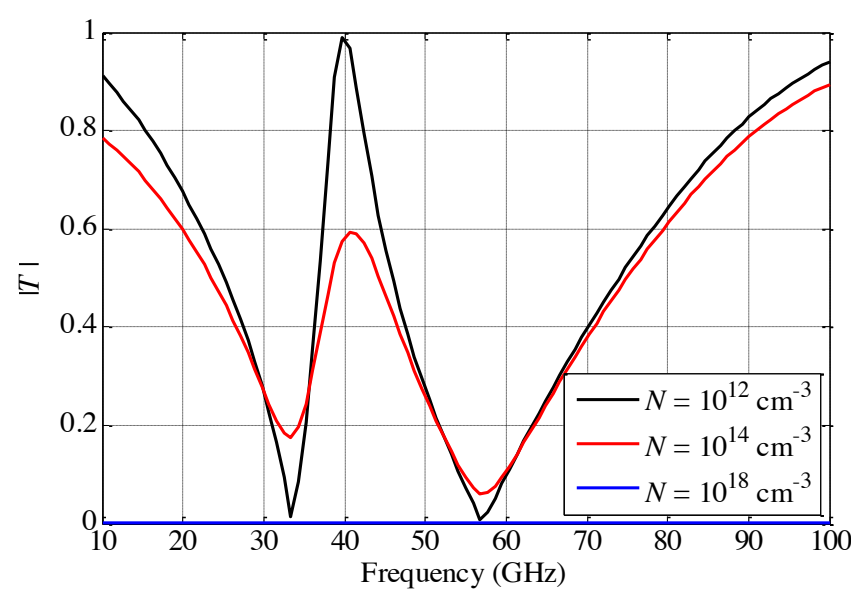

(b)

Figure 7. Reflection (a) and transmission (b) spectra of the array placed on an optically activated silicon substrate for different values of the plasma density $N$.
Remarkably, when the magnetic field strength inside the AF film increases both the real and imaginary parts of magnetic permeability undergo changes. Under a certain threshold level of the input light intensity, this can lead to dispersion-absorption bistable behavior of the system. Here the way to obtain large magnitude of the magnetic field strength in the AF film lies in the choice of the parameters of the DR array of the metamaterial to tune the frequency of the trapped-mode excitation to be close to the frequency of antiferromagnetic resonance. Such a situation is illustrated in Fig. 9 where the optical response of the metamaterial in the case when the intensity of input light is small (linear regime) is presented.

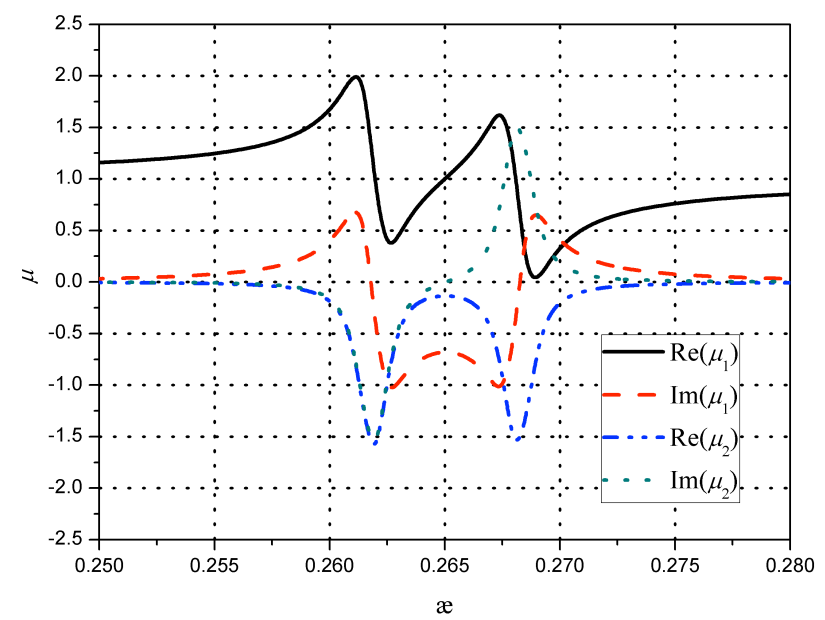

Figure 8. Dispersion curves of the permeability tensor components of the antiferromagnetic film for $\mathbf{H}_{0}=1.0 \mathrm{kG}$.

The behavior of the dispersion characteristics of the AF permeability leads to appearing alternate bands of high transmission and absorption in the spectra of metamaterial. One can see that in the frequency band of the trapped-mode excitation there is a peak of current magnitude, but its frequency dependence has a form of alternating maxima and minima due to the strong absorption in the substrate in the vicinity of the AF resonances nearly $æ_{1} \approx 0.262$ and $æ_{2}$ $\approx 0.268$. However, since the magnetic field strength is proportional to the current and the current magnitude obviously increases when the intensity of the incident field rises, under a certain intensity of the incident field the magnetic properties of substrate can change.

To study such nonlinear response of metamaterial we use the technique proposed in our previous publications $[3,4]$. According to this method, at the first stage, the current distribution $\mathrm{J}$ along the strips, the reflection $\mathrm{R}$ and transmission $\mathrm{T}$ coefficients are determined under the assumption that the intensity of input light $S$ is small (linear regime). For these calculations the Method of Moments is used. At the second stage, the transmission line theory is applied to estimate the inner magnetic field strength which is directly proportional to the current magnitude averaged over a metal pattern. It gives a nonlinear equation related to the average current magnitude in the metal strips. In this equation the input field intensity is a parameter and, at a 
fixed frequency $\omega$, its solution is the average current magnitude which depends on the intensity of the incident field. On the basis of the found current, the inner magnetic field strength is estimated and then the actual parameters of permeability tensor, reflection and transmission coefficients are calculated as functions of the frequency and intensity of the incident field.

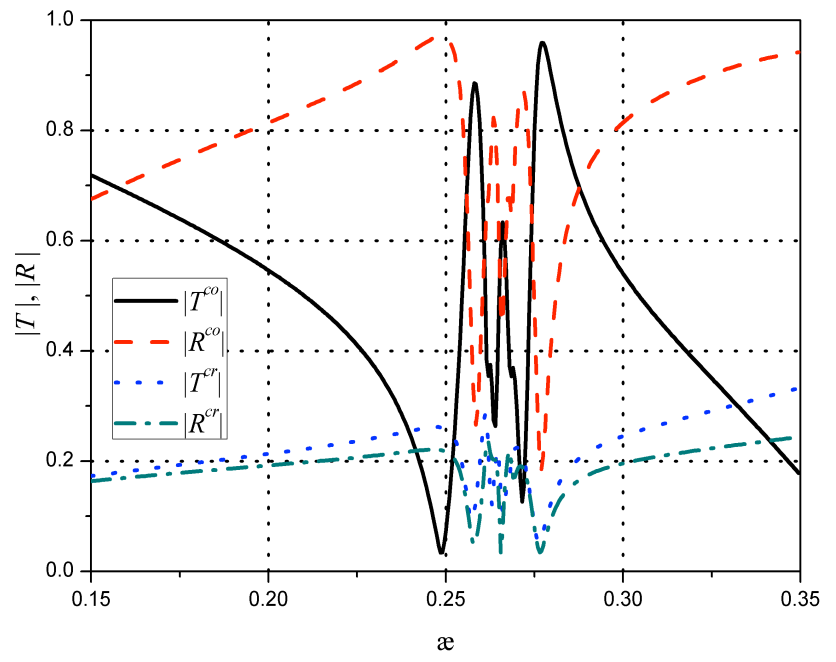

(a)

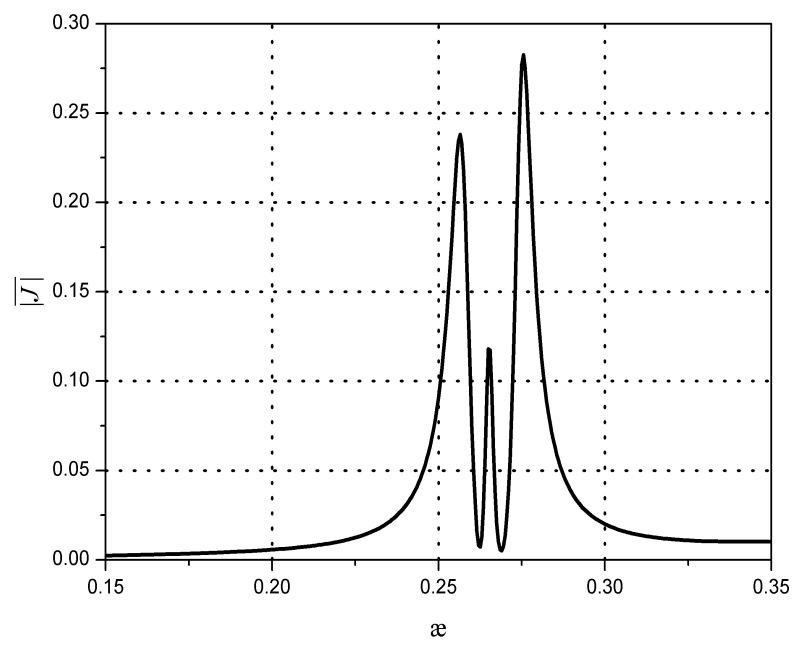

(b)

Figure 9. Reflection, transmission spectra (a) and average current magnitude (b) of the array placed on an antiferromagnetic substrate, linear regime.

Our calculations show that in case of the nonlinear permeability of substrate, dependences of the inner field intensity versus the incident field intensity have a form of hysteresis (Fig. 10a). Such form of curves is studied quite well and is explained by the nonlinear phase-shift and nonlinear attenuation which appear in the nonlinear system. As the incident field intensity increases, the nonlinear phase shift rapidly raises and the attenuation decreases that guarantee the presence of obvious bistable switching.
The frequency dependences of the transmission coefficient magnitudes also manifest discontinuous switching with frequency changing. Since the dispersion curves of nonlinear susceptibility have the bands of grow and decay, the real and imaginary parts of the permeability tensor coefficients also increase and decrease with frequency. The bistable transmission occurs exactly in these frequency bands and is manifested in the ambiguity of the transmission coefficient magnitudes at the leading and trailing edges of the resonance.

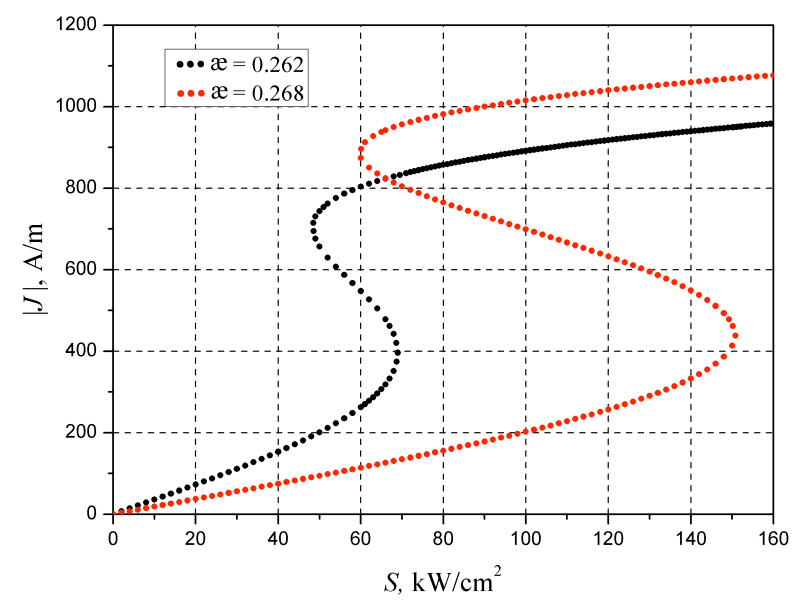

(a)

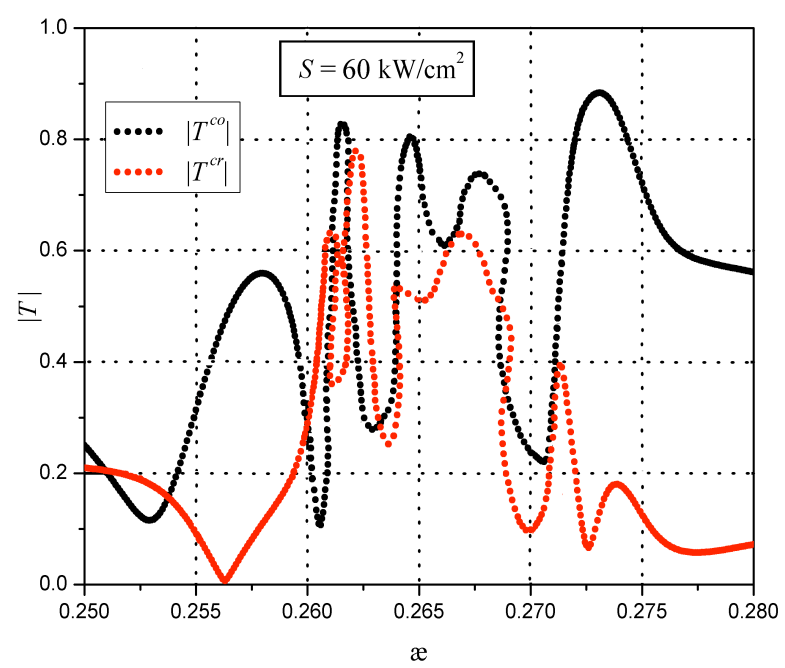

(b)

Figure 10. Inner field intensity versus incident field intencity (a) and transmission spectrum (b) of the array placed on an antiferromagnetic substrate, nonlinear regime.

\section{Conclusions}

We presented electromagnetically controllable surfaces designed on the basis of periodic DR metallization arrays which are placed on a thin substrate of active material. The DR metallization array has the interesting peculiarity of 
supporting the trapped-mode resonance with polarization insensitivity feature for a normally incident electromagnetic plane wave. Using a magnetized ferrite substrate as controlling element, we have shown the possibility to shift the trapped-mode resonant frequency in about $20 \%$, without significant degradation of the transmission band.

An enhancement of Faraday effect was observed in the studied structure. Also by tuning the ferromagnetic resonant frequency to be near the trapped-mode frequency, it is possible to obtain a switch off and on functionality. In the presented configuration the maximum transmittance has a switch off and on ratio of about 0.3 , but we think that with future optimization of the geometrical and physical parameters it is possible to achieve a more complete switching. By using an optically activated silicon substrate as controlling element, we have demonstrated the possibility to have an almost total switching functionality of the transmission band.

We have also shown that in the DR array placed on an antiferromagnetic substrate, the all-optical switching can be realized. It is possible due to the capability of studied metamaterial provides the sufficient field localization inside the thin nonlinear substrate at the frequency of the trappedmode excitation. In conclusion, a planar DR metamaterial, which bears a high-quality-factor trapped-mode resonance, is a promising object for realization of a polarizationinsensitive controllable switching.

\section{Acknowledgements}

We would like to acknowledge the Brazilian agencies CAPES and CNPq, and National Academy of Sciences of Ukraine (Program "Nanotechnologies and Nanomaterials", Project no. 1.1.3.17).

\section{References}

[1] S. Prosvirnin and V. Dmitriev, Electromagnetic wave diffraction by array of complex-shaped metal elements placed on ferromagnetic substrate, European Physical Journal, Applied Physics, vol. 49, 2010.

[2] M. Kawakatsu, V. Dmitriev, and S. Prosvirnin, Microwave Frequency Selective Surfaces with High QFactor Resonance and Polarization Insensitivity, Journal of Electromagnetic Waves and Applications, vol. 24, no. 2-3, pp. 261-270, 2010.

[3] V. Tuz, S. Prosvirnin, and L. Kochetova, Optical bistability involving planar metamaterials with broken structural symmetry, Physical Review B, vol. 82, P.233402, 2010.

[4] V. Tuz, S. Prosvirnin, All-optical switching in metamaterial with high structural symmetry - bistable response of nonlinear double-ring planar metamaterial, European Physical Journal, Applied Physics, vol. 56, 30401, 2011.

[5] R. Mittra, C.H. Chan and T. Cwik, Techniques for analyzing frequency selective surfaces - A Review, Proceedings of the IEEE, pp. 1593-1615, 1988.

[6] B. Lin, S. Liu and N. Yuan, Electrically and magnetically anisotropic substrates, IEEE Transactions on Antennas and Propagation, vol. 54, 2006.

[7] www.cst.com.

[8] D.M. Pozar, Microwave Engineering, John Wiley and Sons, New York, 1998.

[9] C.H. Lee, P.S. Mak, and A.P. De Fonzo, Optical control of millimeter-wave propagation in dielectric waveguides, IEEE Journal of Quantum Electronics, vol. $16,1980$.

[10] S.-C. Lim, J. Osman and D. Tilley, Calculation of nonlinear magnetic susceptibility tensors for a uniaxial antiferromagnet, Journal of Physics D: Applied Physics, vol. 33, pp. 2899-2910, 2000.

[11] Y. Zhao, S.-F. Fu, H. Li, and X.-Z. Wang, Bistable transmission of antiferromagnetic Fabri-Perot resonator, Journal of Applied Physics, vol. 110, 023512, 2011. 